\title{
Squamation of Larval Greenling Hexagrammos otakii (Pices: Hexagrammidae) Reared in the Laboratory
}

\author{
Osamu FukUHARA*1 and Toru Fushimi*2 \\ (Accepted September 1, 1983)
}

\begin{abstract}
Early squamation of Hexagrammos otakii was observed on approximately 100 laboratoryreared specimens ranging from 20 to $56 \mathrm{~mm}$ in standard length (SL). Alizarin staining was used to observe the appearance of scales on the body of different larval stages.

Scales first appeared on larvae 27 to $32 \mathrm{~mm}$ SL on the anterior portion of the trunk and midlaterally on the caudal peduncle. Sequence of squamation is illustrated and figured. Fully squamated specimens were longer than $53.0 \mathrm{~mm}$ in standard length.
\end{abstract}

Morphological development during larval to young stages have been reported previously in detail using laboratory reared specimens." ${ }^{1)}$ Fragmental morphological observations of wildcaught specimens have been described by several authors. ${ }^{2-5)}$ The knowledges on squamation and body size relation of the greenling Hexagrammos otakii is lacking. Biological characteristics of early developmental stages are requisite for understanding the life history in the wild as well as for rearing fingerlings for aquaculture purposes.

In this paper we describe squamation during development.

\section{Materials and Methods}

Specimens used in this study were nearly equivalent to those in the previous paper. ${ }^{1)}$ Alizarin staining was used on the specimens to observe scale formation as it related to larval size. The skin of the left side was removed for observing squamation under a dissecting and/or large-power microscope. Fish size was measured by a profile projector at $10 \times$ for smaller individuals with a precision of $0.1 \mathrm{~mm}$, and calipers were used for longer ones. Abnormally-shaped specimens were excluded from the study.

\section{Results and Discussion}

The sequence of scale formation is shown diagrammatically in Fig. 1 as depicted by nine stages (A-I) of development. Each stage was identified by its extent of scale coverage on the body. The squamated area was differentiated by two types: one was the area squamated by scatter or loose forming scales, and the other was the area squamated by tightly fitting scales with ctenii (Fig. 2). Each developmental stage was characterized as:

A, few scatter scales first appeared simultaneously on the anterior end of the trunk and midlaterally on the caudal peduncle.

$B$, the squamated area of scatter scales extended anteriorly along the lateral line. Small patches of scatter scales were seen at the dorso- and ventrosurface of the caudal peduncle.

$C$, the squamated area of scatter scales extended ventrally gradually, and a few scales with ctenii first appeared on the anterior portion of the caudal peduncle.

D, scatter scales extended dorso-ventrally. The caudal peduncle was completely covered, as was the base of the anal fin. The scales with ctenii extended anterior to the vertical of the spinous rays of the dorsal fin.

E, the caudal area almost covered with scatter scales or scales with ctenii except for the small area above the anal fin. The scales with ctenii developed toward the head, reaching the operculum.

$\mathrm{F}$, the unsquamated areas were small patches below the dorsal and above the ventral fin. The caudal was almost completely covered with ctenii scales, except for the base of the unpaired fins.

$\mathrm{G}$, the only area devoid of sclaes was around the base of the pectoral fin. Scales with ctenii were

*1 Nansei Regional Fisheries Research Laboratory, Ohno, Hiroshima 739-04, Japan（福原 修：南酉海区 水産研究所).

*2 Hiroshima-Ken Saibai Gyogyou Kyokai, Takasaki Takehara, Hiroshima 729-23, Japan（伏見 徽：広 島県载培源策鿍会)。 


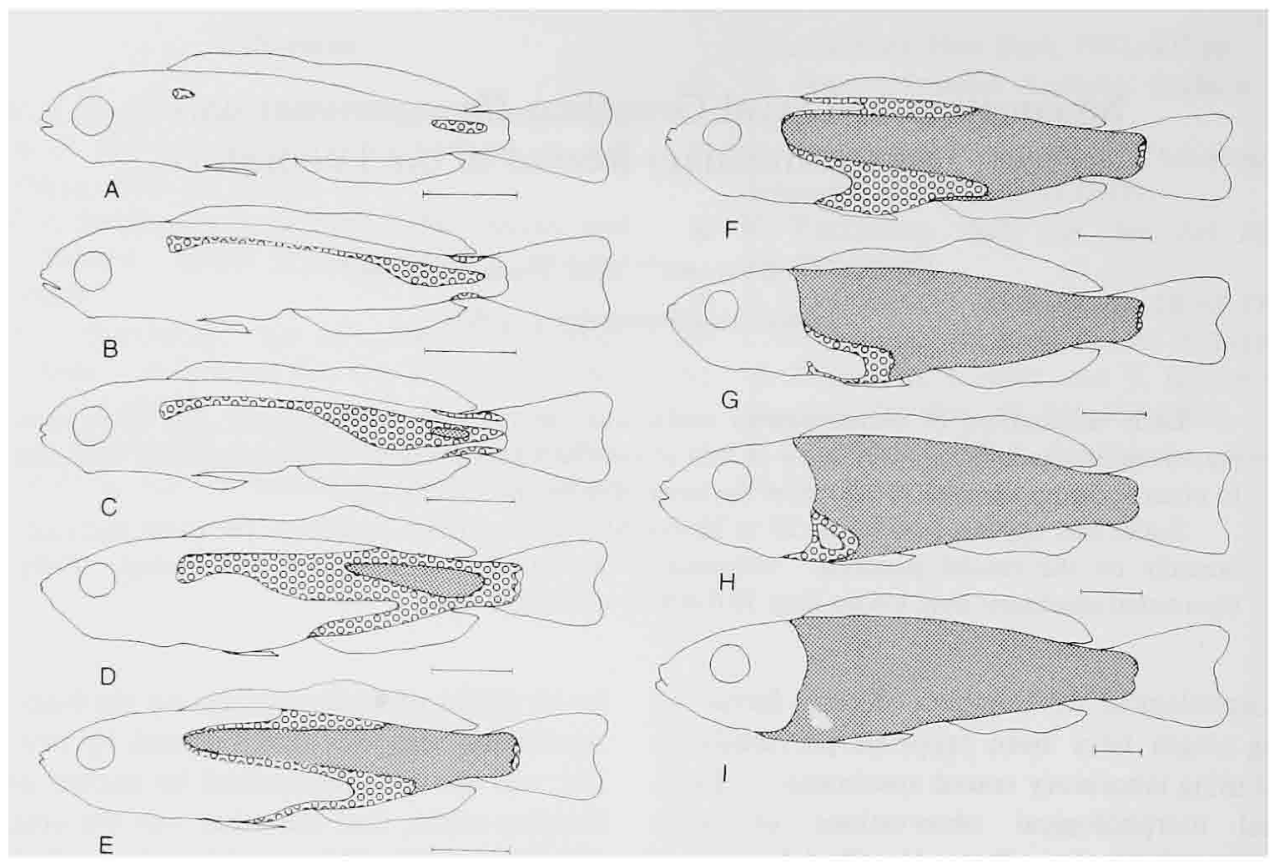

Fig. 1. Sequence of the squamation of larval Hexagrammos otakii reared in the laboratory. Open circles and dotts indicate squamated areas of scatter scales and ctenoid scales, respectively. Refer to Fig. 2 for the detail of extent in squamation. Scales denote $5.0 \mathrm{~mm}$.
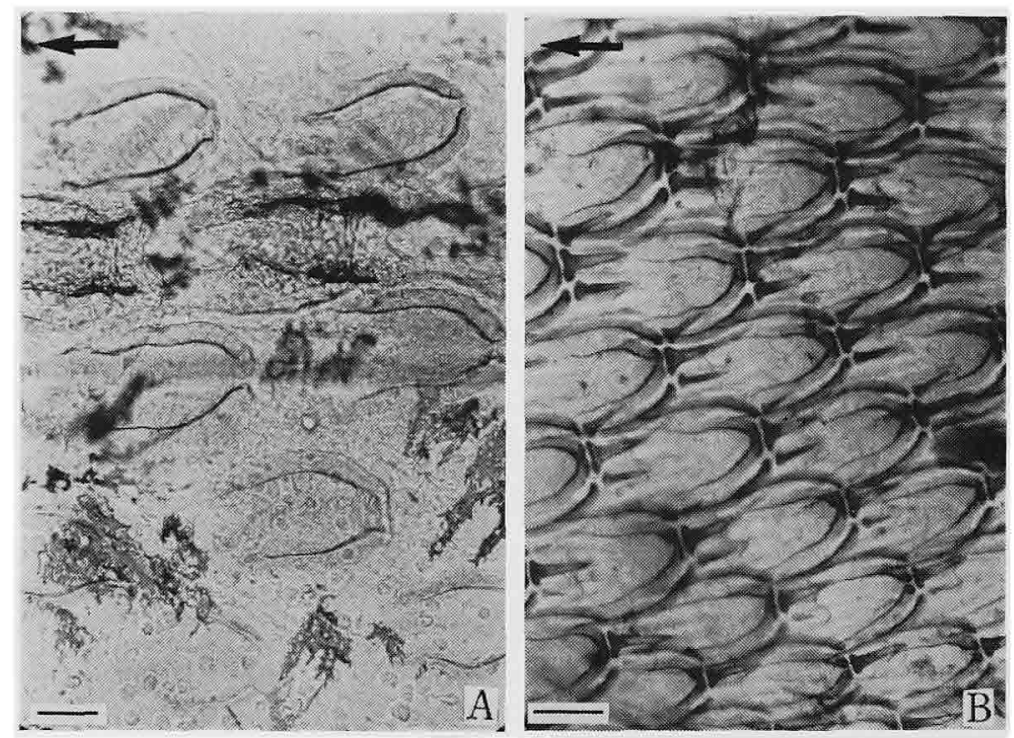

Fig. 2. An example of squamation on the body surface of Hexagrammos otakii. A, scatter scales; B, scales with ctenii and tightly formed. Arrows indicate the head direction. Scales, $100 \mu \mathrm{m}, \times 40$.

not seen only at the base of the caudal fin and along the abdomen.

$\mathrm{H}$, the body surface was covered with ctenii scales except for a small area around the pectoral fin.

I, completely squamated.
Fig. 3 depicts the relationship between the developmental stages and larval size of standard length. The largest specimen without scales was $29.0 \mathrm{~mm}$ SL. Squamation commenced when fish were 27.0 to $32.0 \mathrm{~mm}$ SL., and proceeded as they grew. The smallest specimen fully squamated was 


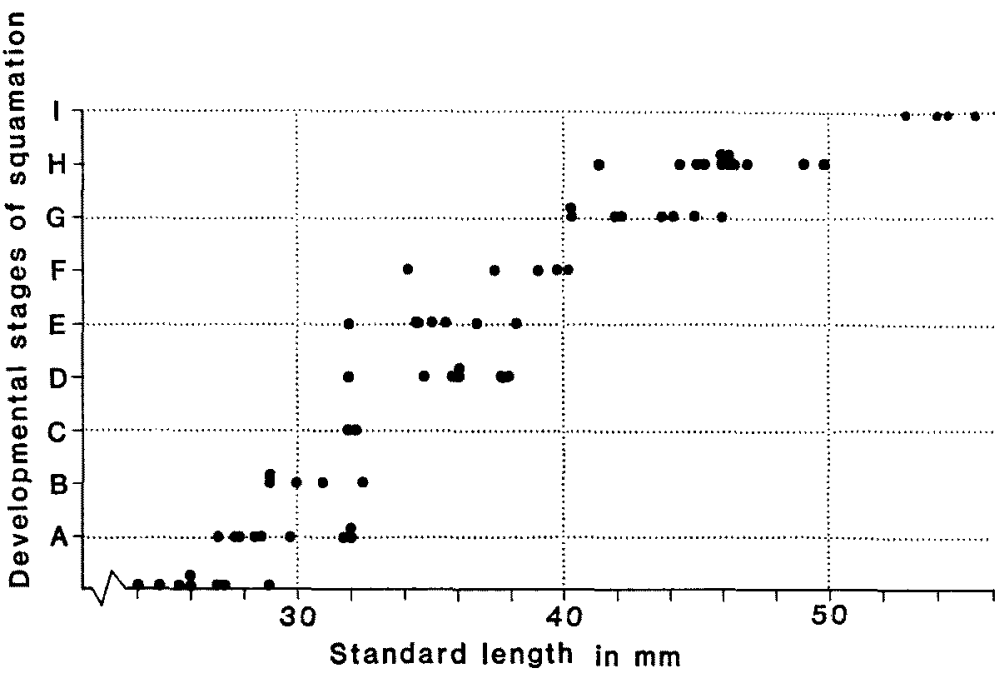

Fig. 3. Developmental stages of scale formation plotted against standard length for larval $\mathrm{Hexa}$ grammos otakii. Developmental stages are identical to that in Fig. 1. Refer to the text for details of the developmental stages.

a larva $53.0 \mathrm{~mm}$ SL. From these observations, it is obvious that scale formation occurred during larval development on fish 27.0 to $53.0 \mathrm{~mm}$ SL. It was assumed that the period of squamation corresponded to fish ages of about 70 to 80 days after hatching based on the growth curve for reared specimens.

No comparison of squamation was available between wild-caught specimens and reared individuals due to a lack of squamation information on the wild fish. In rearing experiments, ${ }^{8,7)}$ fish changed body colour from greenish to brownish when they attained length of 45 to $50 \mathrm{~mm}$. The change of body colour is assumed to related to scale formation. It is also believed that body colour has the role of concealing the fish in their habitats ${ }^{8-10)}$ and scales protect the body surface from various changes and stimuli in environmental conditions. ${ }^{11-13)}$ Accordingly, juveniles, squamated completely, of more than approximately $50 \mathrm{~mm} \mathrm{SL}$ and 80 days old after hatching, are presumably capable of enduring wider ranges of environmental changes than they were before.

\section{Acknowledgements}

The authors express their sincere thanks to Professor T. IwaI, Kyoto University, for the suggestion and advice for the manuscript. Thanks also due to Dr. C. S. MANoOCH III of Southeast Fisheries Center, Beaufort Laboratory for his critical reading of the manuscript.

\section{References}

1) O. Fukuhara and T. Fushimi: Bull. Japan. Soc. Sci. Fish., 49, 1843-1848 (1983).

2) N. N. Gorbunova: Greenlings (Translated from Russian by M. RAver). Israel Program for Scientific Translations, Jerusalem, 1970, pp. 121185.

3) S. Mito: Japan. Marine Plankton, No. 7, Fish Eggs and Larvae. Soyosha, Tokyo, 1966, pp. 6465.

4) H. NaKamura: Youshoku Kaishi, 6, 133-139 (1936).

5) Y. Oshima and N. NaKamura: Suisan Gakaiho, 9, 81-89 (1944).

6) K. TANGE: Saibai Giken (Tech. Rep. Farm. Fish.)., 9, 31-38 (1980).

7) M. Yamada, H. Keitoku, and H. Mizukure: Ann. Rep. Hiroshima Pref. Farm. Center., 1981, 58-64 (1982).

8) M.H. A. Keenleyside: Diversity and Adaptation in Fish Behaviour, Springer-Verlag, Berlin, 1979, pp. 44-62.

9) J. R. Norman: in "A History of Fishes" (ed. by P. H. Greenwood), 3rd ed., Ernest Benn Limited, London, 1975, pp. 195-216.

10) K. UCHIDA: in "The Embryology of Vertebrate" (ed. by M. KUME), Baifukan, Tokyo, 1966, pp. 115-122.

11) N.Y. Kawamoto: Fish Physiology, 1st ed., Ishizaki-syoten, 1956, Tokyo, pp. 211-228.

12) K. Matsubara: in "Systematic Zoology of the Animal Kingdom" (ed. by I. UCHIDA), Vol. 9, Nakayama-syoten, Tokyo, 1963, p. 116.

13) J. V. Oosten: in "The Physiology of Fishes" (ed. by M. E. Brown), Vol. 1, Academic Press, New York, 1957, pp. 207-244. 